\title{
The maths behind combination therapy
}

Therapies targeted at specific genetic aberrations in tumours are increasingly being recognized as successful only in the short term. The heterogeneity of solid tumours means that resistant clones emerge and the disease progresses. Combination therapy holds out hope of limiting this phenomenon, and now a mathematical model has shown that concurrent combination therapy is indeed more potent than single therapy or sequential combination treatment.

Ivana Bozic, first author of the paper, explains the methods used: "we used clinical data from 20 patients with melanoma receiving the BRAF inhibitor vemurafenib to obtain parameters needed for modelling, and a different dataset of patients with metastatic pancreatic, colorectal and melanoma cancer to estimate the numbers and sizes of metastatic lesions in typical candidates for clinical trials. We used a stochastic, branching process model to describe the evolution of resistance to individual drugs and combinations in metastatic lesions."
Their model—which should be applicable to any solid tumourdemonstrated that if there is even a single point mutation that could confer resistance to both drugs, then combination treatments will not be curative in most patients with advanced-stage disease. Furthermore, the treatment protocol is important, as Bozic explains, the model shows that "administering one drug and then the other is a certain recipe for treatment failure, even in situations when the same drugs given simultaneously would cure almost everyone."

These data clearly support concurrent combination treatment with targeted agents for all future clinical trials.

\section{Rebecca Kirk}

Original article Bozic, I. et al. Evolutionary dynamics of cancer in response to targeted combination therapy. eLife 2, e00747 (2013)

Further reading Hutchinson, L. Gastrointestinal cancer: a step closer to combating acquired resistance in CRC. Nat. Rev. Clin. Oncol. 9, 428 (2012) 